\title{
Urinary Angiotensinogen-Melatonin Ratio in Gestational Diabetes and Preeclampsia
}

\author{
Gabriela Ribeiro Valias ${ }^{1}$, Patricia Rodrigues Lourenço Gomes ${ }^{2}$, Fernanda G. Amaral ${ }^{3}$, \\ Saif Alnuaimi ${ }^{4}$, Daniela Monteiro ${ }^{1}$, Siobhán O'Sullivan ${ }^{5}$, Renato Zangaro ${ }^{1}$, \\ José Cipolla-Neto ${ }^{2}$, Juan Acuna ${ }^{4}$, Ovidiu Constantin Baltatu ${ }^{1,4 *}$ and \\ Luciana Aparecida Campos ${ }^{1,4 *}$
}

${ }^{1}$ Center of Innovation, Technology and Education (CITE) at Anhembi Morumbi University-Anima Institute, Sao Jose dos Campos Technology Park, Sao Jose dos Campos, Brazil, ${ }^{2}$ Department of Physiology and Biophysics, Institute of Biomedical Sciences, University of São Paulo, São Paulo, Brazil, ${ }^{3}$ Department of Physiology, Federal University of São Paulo, São Paulo, Brazil, ${ }^{4}$ Department of Public Health and Epidemiology, Khalifa University, Abu Dhabi, United Arab Emirates, ${ }^{5}$ Department of Molecular Biology and Genetics, Khalifa University, Abu Dhabi, United Arab Emirates

OPEN ACCESS

Edited by:

Jingyan Han,

Boston University, United States

Reviewed by:

Arianna Vignini,

Marche Polytechnic University, Italy

Miriam Zacchia,

University of Campania Luigi Vanvitelli,

Italy

*Correspondence: Ovidiu Constantin Baltatu ocbaltatu@gmail.com Luciana Aparecida Campos camposbaltatu@gmail.com

Specialty section: This article was submitted to Nanobiotechnology,

a section of the journa

Frontiers in Molecular Biosciences

Received: 23 October 2021

Accepted: 04 February 2022

Published: 02 March 2022

Citation:

Valias GR, Gomes PRL, Amaral FG, Alnuaimi $S$, Monteiro $D$, O'Sullivan $S$, Zangaro R, Cipolla-Neto J, Acuna J, Baltatu OC and Campos LA (2022) Urinary Angiotensinogen-Melatonin Ratio in Gestational Diabetes and Preeclampsia.

Front. Mol. Biosci. 9:800638. doi: 10.3389/fmolb.2022.800638
Background: A large research portfolio indicates that an activated renal renin-angiotensin system or a deficit on melatonin is associated with several cardiovascular pathologies. In this observational clinical study, we hypothesized that alterations in urinary melatonin or angiotensinogen levels may be altered in two common conditions, preeclampsia and gestational diabetes. Our study's primary objective was to assess melatonin and angiotensinogen as novel disease biomarkers detectable and quantifiable in the urine of pregnant women with or without pregnancy complications.

Methods: This was a concurrent cohort study of pregnant women with selected obstetric pathologies (gestational diabetes, preeclampsia, hypertension and obesity with hypertension). A group of healthy controls was also included. Urinary 6sulfatoxymelatonin and angiotensinogen were measured by sensitive and specific ELISAs in first morning void urine samples. The patients were included in the cohort consecutively, and the diagnosis was blinded at the level of urine collection. Urinary 6sulfatoxymelatonin and angiotensinogen levels were investigated in the patients included in the cohort.

Results: Urinary levels of angiotensinogen were significantly higher in the gestational diabetes [angiotensinogen/creatinine ratio median (25th, 75th): $0.11(0.07,0.18)]$ and preeclampsia $[0.08(0.06,0.18)]$ groups than in those with healthy pregnancy $[0.05(0.04$, 0.06]; 6-sulfatoxymelatonin levels were significantly lower in the gestational diabetes [ug/h: median (25th, 75th): $0.12(0.08,0.17)]$ and preeclampsia $[0.12(0.09,0.15)]$ groups than in those with healthy pregnancy $[0.20(0.15,0.27]$. Neither morning void protein/creatinine ratio nor 24-h urine protein estimate were significantly different between the study groups.

Conclusion: These results suggest that urinary angiotensinogen levels may indicate an intrarenal RAS activation while melatonin production appears to be defective in gestational diabetes or hypertension. An angiotensinogen/melatonin ratio is suggested as an early biomarker for identification of gestational diabetes or hypertension. This report provides a 
basis for the potential use of melatonin for the treatment of preeclampsia. A prospective study in a larger number of patients to determine the operative characteristics of these markers as potential diagnostic tests is justified.

Keywords: angiotensinogen, melatonin, preeclampsia, gestational diabetes, biomarker

\section{INTRODUCTION}

Preeclampsia and gestational diabetes mellitus are two common complications of pregnancy including cerebral and cardiac incidents, multiorgan failure such as end-stage renal disease (ESRD) and are the major causes of perinatal morbidity and mortality where early identification of those at risk is highly beneficial (Vadalà et al., 2017; Swiątkowska-Stodulska et al., 2018). Management for these conditions has been primarily aimed at symptoms that occur late in the course of the disorder. Women who develop preeclampsia during pregnancy are also at increased risk of eclampsia and cardiovascular events, both in the short and long term (Fishel Bartal and Sibai, 2020). Gestational diabetes prevalence rises with increased pregnancy age, maternal obesity and inactivity (Farrar, 2016). Also, there is a lack of consensus between healthcare professionals on methods of screening for gestational diabetes mellitus (Sert and Ozgu-Erdinc, 2021). Present research efforts are primarily focused on the advancement of early detection and therapeutic strategies. Identification of accurate biochemical markers for predicting and early diagnosing complications of pregnancy may have a major effect on maternal and fetal health.

Several lines of evidence have shown the interaction and role of the renin-angiotensin and melatonin systems in the pathways of several cardiovascular pathologies (Campos et al., 2013). Scientific evidence indicates the involvement of reninangiotensin-aldosterone in pregnancy-induced hypertension and diabetes etiopathogenesis (Świątkowska-Stodulska et al., 2018). Urinary angiotensinogen, AT1 receptor antibodies, plasma renin and prorenin, maternal circulatory angiotensin II, and gene polymorphisms have been implicated (Gathiram and Moodley, 2020). Urinary angiotensinogen, a precursor to the renin-angiotensin system, has been identified by us and others as a potential early marker of diabetic nephropathy and chronic kidney disease (Kobori and Navar, 2011; de Alencar Franco Costa et al., 2015). A recent cross-sectional study showed that urinary angiotensinogen/creatinine ratio was correlated with high blood pressure and proteinuria in preeclampsia (Yilmaz et al., 2015). The renin-angiotensin system has also been implicated in the pathogenesis of preeclampsia (Verdonk et al., 2014; Blois et al., 2015). However, the results of investigations on the significance of the renin-angiotensin system in gestational diabetes and preeclampsia have been contentious (Gathiram and Moodley, 2020).

Melatonin, a circadian pineal hormone with free radical scavenging and antioxidant effects, has been investigated extensively in patients with cardiovascular disease and kidney injury (Baltatu et al., 2017). Emerging evidence supports melatonin in the pathophysiology of pregnancy and fetal development (Tain et al., 2017). Melatonin was found to be associated with maternal plasma antioxidant status in the first trimester of pregnancy and to be deficient in the development of obstetric complications (Ramiro-Cortijo et al., 2016). It has also been proposed that the decrease of circulating melatonin levels could be correlated with the development of preeclampsia. (Zeng et al., 2016). Significant effects of melatonin in placenta have been demonstrated in pre-eclampsia physiopathology (SagrilloFagundes et al., 2014). A systematic review indicated a relationship between melatonin receptor $1 \mathrm{~B}$ and insulin receptor substrate 1 polymorphisms associated with an increased risk of developing gestational diabetes mellitus (Zhang et al., 2014). Newly, melatonin synthesis impairment is considered as a deleterious outcome of diabetes-derived hyperglycemia (Campos et al., 2013; Amaral et al., 2014).

In addition to proteinuria, which is the most prevalent test during pregnancy (Fishel Bartal et al., 2020), novel urine biomarkers for early diagnosis of disorders that complicate pregnancy are being proposed (Aitekenov et al., 2021). Based on the evidence presented, we here hypothesized that alterations in urinary melatonin or angiotensinogen can occur in early stages of gestational diabetes or hypertension and that these biomarkers may potentially be helpful in the prediction and early identification of patients with both preeclampsia and gestational diabetes. Therefore, the primary objective of our study was to assess melatonin and angiotensinogen as potential novel biomarkers of disease that are present and quantifiable in the urine of pregnant women. In addition, we investigated the potential synergy of combined biomarkers strategy for pregnancy complications.

\section{METHODS}

This is a concurrent cohort study using consecutive sampling. All patients were recruited from the prenatal care system of a single institution and included in the cohort based on inclusion criteria and consent to participate in the study. The subjects included were then followed up for the development of pregnancy complications (Figure 1). A subset of the cohort corresponded to normal patients. Biomarkers were assessed and their levels studied for association to the final presence or absence of disease complications in the pregnancies. The study sample size was estimated based on a prior study in which the standard deviation of the main variable (urinary angiotensinogen/creatinine ratio) was 2.1, detecting significant differences between three groups of study participants totaling 90 (Yilmaz et al., 2015). The probability is 80 percent that the study will detect a difference between groups of study at a two-sided 0.05 significance level, if the true difference between treatments is 1.2 units. The calculations led to a total of 100 patients to be recruited in this study. Considering $10 \%$ 


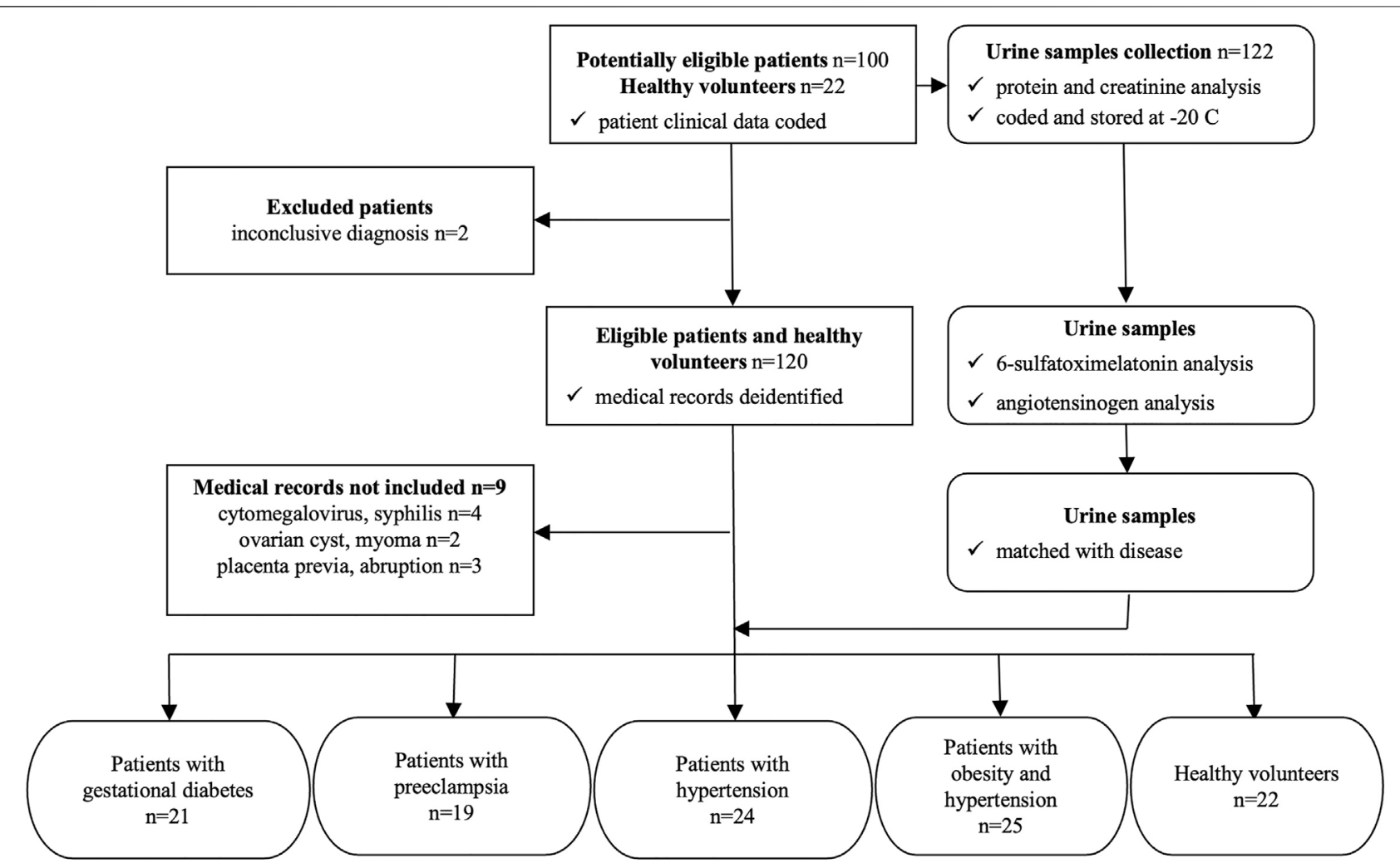

FIGURE 1 | STARD diagram to report flow of participants through the study.

dropouts due to various factors, we calculated to recruit approximately 110 pregnant women in the study. Also, since this is a cohort observational model of the study, we expected that we would recruit in 1 year at least 110 pregnant women, as estimated by Hospital São Francisco de Assis - Jacareí.

The study was approved by the Ethical Committees of the Anhembi Morumbi University (CAAE 79831417.7.0000.5492), University of Sao Paulo (CAAE 30460114.5.0000.0068) and Hospital São Francisco de Assis. It was conducted in accordance with International Council for Harmonization of Technical Requirements for Pharmaceuticals for Human Use Guidelines and the Declaration of Helsinki. All patients provided written informed consent before entry into the study.

\section{Patient Selection}

Pregnant women attending prenatal care at Sao Francisco de Assis Hospital, Jacareí/Sao Paulo were invited to participate to the study (During ---period of time of the recruitment--). Their selection was consecutive at the time of admission, and the diagnosis was blind to the level of urine collection and testing for the biomarkers. One hundred and 22 potentially eligible women were recruited that included 100 patients with gestational diabetes and/or preeclampsia, and 22 pregnant women without complications. Although this was not a diagnostic accuracy study, a STARD diagram was modified to display the flow of participants in the study (Figure 1) (Bossuyt et al., 2015). From the 122 study participants, first morning void urine samples were collected for protein and creatinine measurements, after which were aliquoted in duplicates, coded and stored at $-20^{\circ} \mathrm{C}$. The clinical diagnosis of study participants were coded (potentially eligible patients) and deidentified (eligible patients and healthy volunteers) according to the Brazilian General Data Protection Law (Galvin and DeMuro, 2020). The urine samples were encoded only by numbers and the link between numbers and patient names stored in a password protected computer program, according to the Brazilian General Data Protection Law (Galvin and DeMuro, 2020). After the urinary 6-sulfatoxymelatonin and angiotensinogen measurements, data were matched with the diagnosed diseases of 111 study participants (Figure 1). The characteristics of the study cohort was as follows: ages eligible for study-18 years and older, accepts healthy volunteers-yes, sampling method-nonprobability sample. The following criteria of inclusion and exclusion were applied for the selection and enrollment of pregnant women in the study. Inclusion criteria: gestational age between 6 and 22 weeks for the first visit determined by ultrasound, high risk group (presence of specific risk factors for preterm delivery, pregnancy-induced hypertension or intrauterine growth retardation), low risk group (normal pregnancy with no risk factors for preterm delivery, pregnancy-induced hypertension or intrauterine growth retardation). Exclusion criteria were: known malignancy, pregnancies without ultrasonographic confirmation of gestational age, multifetal pregnancy with greater than or 
equal to 3 fetuses, active vaginal bleeding, serious medical illness (renal insufficiency, congestive heart disease, chronic respiratory insufficiency), asthma requiring systemic steroids, patient requiring anti-platelet or non-steroidal anti-inflammatory drugs, active hepatitis, and lack of consent.

\section{Clinical Evaluations}

The diagnosis of preeclampsia or gestational diabetes were made by the hospital's obstetricians using the following criteria. Preeclampsia was defined as de novo hypertension with high blood pressure (systolic $\geq 140$ or diastolic $\geq 90 \mathrm{~mm} \mathrm{Hg}$ ) on two occasions, at least 4 hours apart, after 20 weeks of pregnancy and proteinuria of $0.3 \mathrm{~g}$ in $24 \mathrm{~h}$ urine collection, or $2+$ in 2 samples with $2 \mathrm{~h}$ apart, or more than $0.3 \mathrm{mg}$ in a single sample (Sousa et al., 2019; Leal et al., 2020). Gestational diabetes mellitus was defined as carbohydrate intolerance, resulting in hyperglycemia of varying severity with onset or first recognition during pregnancy. Development of gestational diabetes at 24-28 weeks of gestation for abnormal values in the $75 \mathrm{~g}$ oral glucose tolerance test (American Diabetes Association, 2013), as recommended by the Brazilian Ministry of Health (Dos Santos et al., 2020). Patients with preexistent hypertension were those diagnosed with hypertension prior or up to the 20th week of pregnancy (Sousa et al., 2019). Obesity was defined by body mass index (BMI) values $\geq 30 \mathrm{~kg} / \mathrm{m}^{2}$ and identified as a condition prior to pregnancy (Ferreira et al., 2019; Indarti et al., 2021). First morning void urine samples were collected for the study at the time of admission after signing the consent form.

\section{Clinical Laboratory, 6-Sulfatoxymelatonin and Angiotensinogen Measurements}

Urine samples were obtained in the event of pregnancy complications, at which time pregnant women sought medical help; urine was collected as the first morning urine, and was used for the protein, creatinine, 6-sulfatoxymelatonin and angiotensinogen urinary tests (Figure 1).

Proteinuria $(\mathrm{mg} / \mathrm{dl})$, urinary creatinine $(\mathrm{mg} / \mathrm{dl})$ and the protein/creatinine ratio were determined by the hospital's laboratory. From these measures, the $\mathrm{mg} / 24 \mathrm{~h}$ ratio of creatinine excretion and the $24-\mathrm{h}$ urinary protein estimate $(\mathrm{mg} / 24 \mathrm{~h})$ were determined using the Fotheringham's et al. equation (Fotheringham et al., 2014).

Urinary 6-sulfatoxymelatonin and angiotensinogen were measured by sensitive and specific ELISAs in first morning void urine samples. All maternal samples were analyzed in duplicate, and laboratory personnel were unaware of the diagnostic status of the study subjects at the time of analysis. Urinary excretion of angiotensinogen was analyzed by sandwich-ELISA assay (Kobori et al., 2009; de Alencar Franco Costa et al., 2015). Melatonin excreted overnight was determined through urinary 6sulfatoxymelatonin levels (Baltatu et al., 2002; Campos et al., 2020). The ELISA kits for both angiotensinogen and 6sulfatoxymelatonin were from IBL International GmbH Germany.

\section{Statistical Analysis}

Data were tested for normality distribution using D'AgostinoPearson normality test (D'agostino, 2017) and Kolmogorov-
Smirnov test with Dallal-Wilkinson-Lilliefors' $p$ value (Dallal and Wilkinson, 1986). Differences between study groups, were assessed with Kruskal-Wallis nonparametric test, followed by a comparison of the mean rank of each group with the mean rank of the control group (healthy pregnant subjects). Multiple testing correction was done by controlling the False Discovery Rate with a two-stage step-up method of Benjamini, Krieger and Yekutieli (Benjamini et al., 2006). All statistical analyses were carried out using GraphPad Prism version 8.1.2 for Mac OS X, GraphPad Software, La Jolla California United States, www.graphpad.com. Differences were considered significant when the probability of a Type I error was lower than $5 \%$ $(p<0.05)$.

\section{RESULTS}

\section{Patient Demographics and Laboratory Data}

The recruited study cohort included patients with gestational diabetes mellitus $(\%, \mathrm{n})(17.2 \%, 21)$, with preeclampsia $(15.6 \%$, $19)$, with preexistent hypertension $(19.7 \%, 24)$, with preexistent obesity and hypertension $(20.5 \%, 25)$, and healthy volunteers (18.0\%, 22).

Table 1 indicates for each study group the age, gestational age, multiparity, previous cesarean delivery or abortion. No statistical differences were observed between the study groups for age or gestational age. The patients' gestational age was: $24-28$ weeks for patients with gestational diabetes mellitus, 20-33 weeks for patients with preeclampsia, and 20-33 weeks for patients with hypertension, with obesity plus hypertension, or healthy volunteers. Multiparity, previous cesarean delivery, or abortion appear to be more prevalent in disease groups than in healthy group. Urine volume $(\mathrm{ml})$, creatinine $(\mathrm{mg} / \mathrm{dl})$, protein/creatinine ratio $(\mathrm{mg} / \mathrm{mg})$ and $24-\mathrm{h}$ urine protein estimate are presented as median and interquartile range (IQR, 25th-75th percentile) per group of study. In gestational diabetes, urine volume was significantly greater and urine creatinine was significantly lower than in healthy control pregnancies. Protein-creatinine ratio was not different between the study groups-Table 1 and Figure 2A. The 24-h urine protein estimate (mg/24h) was not different between the study groups-Table $\mathbf{1}$ and Figure $\mathbf{2 B}$.

\section{Urine 6-Sulfatoxymelatonin and Angiotensinogen}

Urine 6-sulfatoxymelatonin (ug/h) was significantly reduced in the first morning void urine samples of patients with gestational diabetes and preeclampsia-Figure $\mathbf{3 A}$ and Table $\mathbf{1}$. Urinary angiotensinogen (angiotensinogen/creatinine ratio, ug/g) was significantly increased in the first morning void urine samples of patients with gestational diabetes and preeclampsia-Figure 3B and Table 1.

The ratio of angiotensinogen/6-sulfatoxymelatonin was calculated as (angiotensinogen/creatinine ratio, ug/g)/(6sulfatoxymelatonin, $\mathrm{ug} / \mathrm{h}$ ) significantly increased in the first morning void urine samples of patients with gestational diabetes and preeclampsia-Figure 4 and Table 1. 
TABLE 1 | Patient demographics and laboratory urine analyses in the studied cohort.

\begin{tabular}{|c|c|c|c|c|c|}
\hline \multirow[t]{2}{*}{ Parameter median (25th, 75th) } & \multirow{2}{*}{$\begin{array}{l}\text { Healthy } \\
\text { (n = 22) }\end{array}$} & \multirow{2}{*}{$\begin{array}{c}\begin{array}{c}\text { Gestational } \\
\text { diabetes }\end{array} \\
\text { (n= 21) }\end{array}$} & \multirow{2}{*}{$\begin{array}{c}\text { Preeclampsia } \\
(n=19)\end{array}$} & \multirow{2}{*}{$\begin{array}{l}\text { Hypertension } \\
(n=24)\end{array}$} & \multirow[t]{2}{*}{$\begin{array}{l}\text { Hypertension + obesity (n } \\
\text { = 25) }\end{array}$} \\
\hline & & & & & \\
\hline Age (years) & $\begin{array}{l}28.00(24.00 \\
31.50)\end{array}$ & $32.00(27.50,35.00)$ & $\begin{array}{l}29.00(26.00 \\
33.00)\end{array}$ & $\begin{array}{l}32.50(28.50 \\
36.75)\end{array}$ & $32.00(28.50,37.00)$ \\
\hline Gestational age (weeks) & $\begin{array}{l}27.50(23.75 \\
32.00)\end{array}$ & $25.00(24.00,26.50)$ & $\begin{array}{l}29.00(26.00 \\
33.00)\end{array}$ & $\begin{array}{l}30.50(28.00 \\
33.00)\end{array}$ & $31(28.50,33.00)$ \\
\hline Multiparous [N (\%)] & $9(40.9 \%)$ & $20(95.2 \%)$ & 19 (100\%) & $21(87.5 \%)$ & $16(64 \%)$ \\
\hline Previous cesarean delivery [N (\%)] & $3(13.6 \%)$ & $10(47.6 \%)$ & $10(52.6 \%)$ & $11(45.8 \%)$ & $11(44 \%)$ \\
\hline Previous abortion [N (\%)] & $2(9.1 \%)$ & 9 (42.9\%) & $10(52.6 \%)$ & $6(25 \%)$ & $5(20 \%)$ \\
\hline Urine angiotensinogen/creatinine ratio $(\mu \mathrm{g} / \mathrm{g})$ & $0.05(0.04,0.06)$ & $0.11(0.07,0.18) * \star$ & $0.08(0.06,0.18)^{\star}$ & $0.05(0.04,0.06)$ & $0.06(0.05,0.09)$ \\
\hline Urine 6-sulfatoximelatonin ( $\mu \mathrm{g} / \mathrm{h})$ & $0.20(0.15,0.27)$ & $0.12(0.08,0.17)^{\star}$ & $0.12(0.09,0.15)^{\star}$ & $0.19(0.15,0.32)$ & $0.21(0.17,0.30)$ \\
\hline $\begin{array}{l}\text { Urine angiotensinogen/6-sulfatoximelatonin/ } \\
\text { creatinine Ratio }\end{array}$ & $0.27(0.16,0.32)$ & $0.94(0.36,2.06)^{\star \star}$ & $0.72(0.50,1.40)^{\star \star}$ & $0.29(0.15,0.39)$ & $0.31(0.20,0.48)$ \\
\hline Urine volume (ml) & $\begin{array}{l}50.00(40.00 \\
62.50)\end{array}$ & $70.00(55.00,70.00)$ & $\begin{array}{l}50.00(40.00 \\
60.00)\end{array}$ & $\begin{array}{l}57.50(41.25 \\
70.00)\end{array}$ & $50.00(40.00,60.00)$ \\
\hline Urine creatinine (mg/dl) & $\begin{array}{l}93.75(70.63 \\
133.7)\end{array}$ & $\begin{array}{l}57.00(34.00 \\
82.88)^{\star}\end{array}$ & $\begin{array}{c}80.50(34.00 \\
116.5)\end{array}$ & $\begin{array}{c}95.63(59.88 \\
127.4)\end{array}$ & $123.5(76.5,150.0)$ \\
\hline Protein/creatinine Ratio (mg/mg) & $0.08(0.05,0.15)$ & $0.12(0.08,0.19)$ & $0.14(0.05,0.19)$ & $0.10(0.06,0.18)$ & $0.09(0.04,0.12)$ \\
\hline 24-h urine protein estimate & $\begin{array}{c}145.8(121.1 \\
246.2)\end{array}$ & $129.0(68.52,187.4)$ & $\begin{array}{c}155.5(129.0 \\
216.5)\end{array}$ & $\begin{array}{l}128.5(91.87 \\
224.2)\end{array}$ & $133.0(61.81,174.7)$ \\
\hline
\end{tabular}

${ }^{*} p<0.05,{ }^{* *} p<0.005$.

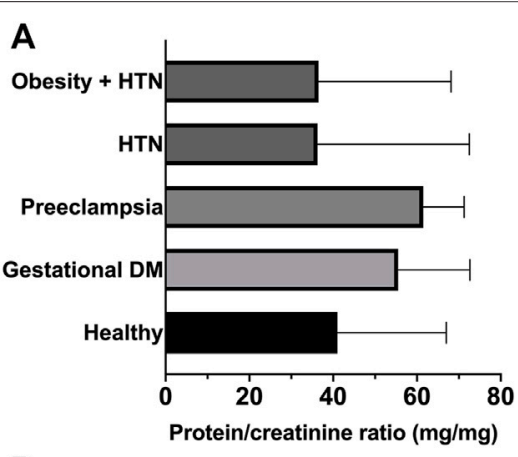

B



FIGURE 2 | (A) Protein/creatinine ratio. (B) 24-h urine protein estimate. Graphs are ranks plots with bars of median with interquartile range; Kruskal-Wallis test, no significant differences between groups.

\section{DISCUSSION}

The main findings of this study are the high angiotensinogen and low melatonin levels in the urine of women with gestational diabetes or preeclampsia. These findings may support the development of diagnostic tools and strategies for recognizing patients at risk of pregnancy complications (Phipps et al., 2016).

Renin-angiotensin system has been involved in the development of preeclampsia. Studies have shown that angiotensinogen, the precursor of all angiotensin peptides ( $\mathrm{Lu}$ et al., 2016), is a particularly good candidate biomarker for both its acute (Ba Aqeel et al., 2017) and chronic (Juretzko et al., 2017) kidney injury (Chen et al., 2016). Research indicates that urinary angiotensinogen comes mainly from the kidney, and higher levels correlate with diabetic albuminuria (Kobori and Navar, 2011; de Alencar Franco Costa et al., 2015). The current study's findings of an elevated urine angiotensinogen/creatinine ratio in preeclampsia corroborate the findings of (Yilmaz et al. (2015) and of Mistry et al. (2019). These findings contrast with those of other research, such as Chen et al. (2011)) or Zhang et al. (2017), which found considerably reduced urine angiotensinogen levels in preeclampsia. Pringle et al. found a rise in urine angiotensinogen/creatinine levels as pregnancy progressed, but a significant decline in the third trimester in a group of women with pregnancy complications that included preeclampsia and gestational diabetes (Pringle et al., 2018). These may imply a time-dependent activation/suppression of the renal reninangiotensin system during preeclampsia, as measured by urine angiotensinogen/creatinine levels. It appears that additional research is necessary to determine the relevance of urinary angiotensinogen in the detection of preeclampsia. Although Pringle et al. included women with gestational diabetes in their investigation, no pathology-specific group analyses were performed on urine angiotensinogen/creatinine levels. As a result, the current study may be the first to report significantly elevated urine angiotensinogen/creatinine levels in gestational diabetes.

A large body of evidence suggests that melatonin has a protective effect during the development of kidney disease, at least in part by counteracting the effects of intrarenal renin- 


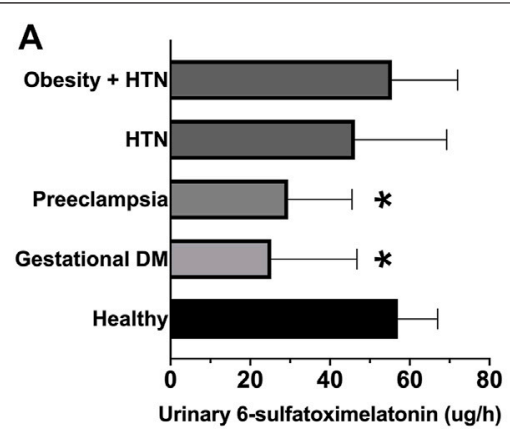

B

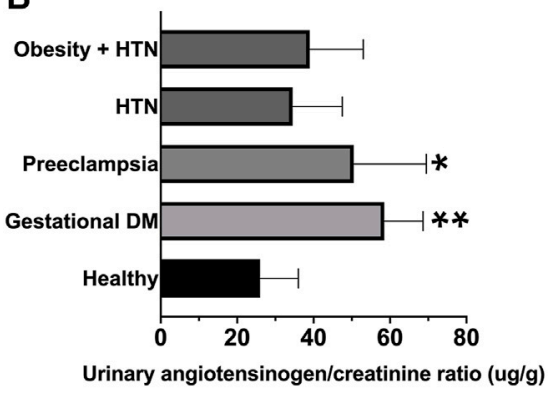

FIGURE 3 | (A) Urinary 6-sulfatoxymelatonin (ug/h) in first morning void urine samples. (B) Urinary angiotensinogen-creatinine ratio (ng/mg). Graphs are ranks plots with bars of median with interquartile range; Kruskal-Wallis test ${ }^{*}, p<0.05$ and ${ }^{* *}, p<0.005$ in comparison to the healthy group.

angiotensin system activation, prompting the need for further research into the roles of melatonin and angiotensinogen in renal injury (Campos et al., 2013; Baltatu et al., 2017; Ohashi et al., 2019). Its role in pregnancy conditions is still to be clearly understood (Tong et al., 2020). The current study's findings of low levels in preeclampsia corroborate Bouchlariotou's et al. findings (Bouchlariotou et al., 2014). Additionally, Bouchlariotou et al. revealed a link between impaired melatonin secretion and an non-dipper blood pressure (no nocturnal physiological blood pressure decrease) in women with preeclampsia. Our study is the first to demonstrate that women with gestational diabetes had decreased urinary 6sulfatoxymelatonin levels. While the findings of Bouchlariotou et al. and ours may not be sufficient to implicate melatonin in the pathogenesis of preeclampsia, they certainly suggest that its participation in the pathogenesis of preeclampsia and gestational diabetes warrants further investigation.

The presence of elevated angiotensinogen and low 6sulfatoxymelatonin in the first morning void urine samples of patients with gestational diabetes or preeclampsia suggests activation of the intrarenal RAS and an impairment in melatonin synthesis. Additionally, the urine angiotensinogen6-sulfatoxymelatonin ratio was elevated in women with gestational diabetes or preeclampsia. Although urine protein/ creatinine levels increased in the gestational diabetes and preeclampsia groups as well, the difference was not statistically significant when compared to the control group. This could be because the study groups included a limited number of cases. This apparent disparity between proteinuria results from the first

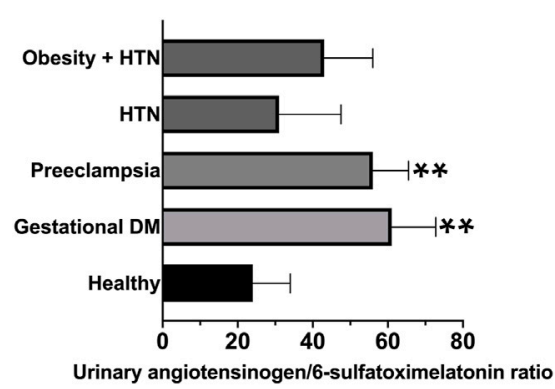

FIGURE 4 | Angiotensinogen/6-sulfatoxymelatonin ratio was calculated as (angiotensinogen/creatinine ratio, $u g / g) /(6$-sulfatoxymelatonin, ug/h). Graphs are ranks plots with bars of median with interquartile range; Kruskal-Wallis test ${ }^{* *}, p<0.005$ in comparison to the healthy group.

morning void and physician diagnosis of preeclampsia could be related to sampling methods, as well. In fact, Lamontagne et al. demonstrated that early morning urine protein was ineffective in detecting preeclampsia (Lamontagne et al., 2014). Although several studies on "spot urine" protein to creatinine ratio estimation suggest a promising diagnostic value for preeclampsia (Stefańska et al., 2020), the test's heterogeneity in accuracy precludes yet its utility as a diagnostic tool (Morris et al., 2012).

There are important limitations of our study that should be recognized. Since our study is cross-sectional, assigning causality between the altered urinary angiotensinogen or melatonin levels and pathogenesis of gestational diabetes and preeclampsia is not possible. Although the recruited cohort might be considered a representative sample for the state of Sao Paulo, this single-center study requires confirmation in various cohorts or multicenter investigations. Although the total number of patients is not insignificant, subgroups of individuals include a limited number of cases, thus the results should be considered as a pilot study requiring further analysis and confirmation. Using multivariable adjusted models, we will be able to assess the impact of other factors on the reported correlations between altered urine angiotensinogen or melatonin levels and gestational diabetes and preeclampsia.

Further longitudinal diagnostic cohort studies are expected to define the operative characteristics of these markers for risk assessment, prediction and diagnosis of the studied conditions. Also, cohort studies may allow to track the pathological evolution of the disease and to explore its potential association with urinary melatonin or angiotensinogen. Further studies need to investigate as well the optimal cutoff value to use urinary angiotensinogen/melatonin ratio as clinical decision guidance.

\section{CONCLUSION}

These findings suggest that the levels of urinary angiotensinogen may indicate intra-renal RAS activation, whereas the production of melatonin appears to be defective in gestational diabetes or 
preeclampsia. For early diagnosis of gestational diabetes or preeclampsia, morning void urine angiotensinogen-melatonincreatinine ratio is suggested. This report provides a rationale for the possible use of melatonin as a potential coadjuvant therapeutic agent for the treatment of preeclampsia or gestational diabetes.

\section{DATA AVAILABILITY STATEMENT}

The datasets generated during and/or analyzed during the current study are available from the corresponding author on reasonable request.

\section{ETHICS STATEMENT}

The studies involving human participants were reviewed and approved by The study was approved by the Ethical Committees of the Anhembi Morumbi University (CAAE 79831417.7.0000.5492), University of Sao Paulo (CAAE 30460114.5.0000.0068) and Hospital São Francisco de Assis. It was conducted in accordance with International Council for Harmonization of Technical Requirements for Pharmaceuticals for Human Use Guidelines and the Declaration of Helsinki. All patients provided written informed consent before entry into the study. The patients/participants provided their written informed consent to participate in this study.

\section{AUTHOR CONTRIBUTIONS}

Conceptualization, LC and OB; methodology, JC-N, PG, FA, RZ; formal analysis, GV, PG, FA, SA, DM, SO'S; investigation, GV, DM; resources, LC, JC-N, OB, JA; data curation, GV, PG,

\section{REFERENCES}

Aitekenov, S., Gaipov, A., and Bukasov, R. (2021). Review: Detection and Quantification of Proteins in Human Urine. Talanta 223, 121718. doi:10. 1016/j.talanta.2020.121718

Amaral, F. G., Turati, A. O., Barone, M., Scialfa, J. H., do Carmo Buonfiglio, D., Peres, R., et al. (2014). Melatonin Synthesis Impairment as a New Deleterious Outcome of Diabetes-Derived Hyperglycemia. J. Pineal Res. 57, 67-79. doi:10. 1111/jpi.12144

American Diabetes Association (2013). Diagnosis and Classification of Diabetes Mellitus. Diabetes Care 36 Suppl 1, S67-S74. doi:10.2337/ dc13-S067

Ba Aqeel, S. H., Batlle, D., and Batlle, D. (2017). Angiotensinogen as a Biomarker of Acute Kidney Injury. Clin. Kidney J. 10, 759-768. doi:10. 1093/ckj/sfx087

Baltatu, O., Afeche, S. C., Santos, S. H. J. d., Campos, L. A., Barbosa, R., Michelini, L. C., et al. (2002). Locally Synthesized Angiotensin Modulates Pineal Melatonin Generation. J. Neurochem. 80, 328-334. doi:10.1046/j.00223042.2001.00701.x

Baltatu, O. C., Amaral, F. G., Campos, L. A., and Cipolla-Neto, J. (2017). Melatonin, Mitochondria and Hypertension. Cell. Mol. Life Sci. 74, 3955-3964. doi:10. 1007/s00018-017-2613-y
FA, DM, SA, SO'S; writing-original draft preparation, LC and OB; writing-review and editing, GV, PG, FA, DM, SA, SO'S, RZ, JC-N, OB, LC, JA; visualization, GV, PG, FA, DM, SA, SO'S, RZ, JC-N, OB, LC; supervision, LC, JC-N, OB; project administration, LC, JC-N, OB, RZ; funding acquisition, LC, JC-N, JA. All authors have read and agreed to the published version of the manuscript.

\section{FUNDING}

This research was supported by Sao Paulo Research Foundation to LC (FAPESP: 2017/11976-0), JC-N (FAPESP 2014/50457-0), Anima Institute - AI (research professor fellowships to $\mathrm{OB}$ an LC), and Khalifa University of Science and Technology under RDISC Award No. 8474000331 to JA. OB and JC-N are supported by the National Council for Scientific and Technological Development (CNPq, 307760/2018-9 and 301324/2018-22).

\section{ACKNOWLEDGMENTS}

We thank the reviewers for their thoughtful review of the manuscript. They raised important issues and their inputs were very helpful for improving the manuscript. We gratefully acknowledge the help and support provided by the colleagues from Sao Francisco de Assis Hospital, Jacareí/ Sao Paulo: obstetrician Marcelo Soares Nogueira (diagnosis), obstetrician Simone Gianneschi (diagnosis), Nrs. Cintia Rodrigues de Oliveira (sample collection and storage), Nrs. Sabrina Sacha de Carvalho Silva (laboratory analysis), Bioméd. Lucimeire Goretti N. Brandão (laboratory analysis).

Benjamini, Y., Krieger, A. M., and Yekutieli, D. (2006). Adaptive Linear Step-Up Procedures that Control the False Discovery Rate. Biometrika 93, 491-507. doi:10.1093/biomet/93.3.491

Blois, S. M., Dechend, R., Barrientos, G., and Staff, A. C. (2015). A Potential Pathophysiological Role for Galectins and the Renin-Angiotensin System in Preeclampsia. Cel. Mol. Life Sci. 72, 39-50. doi:10.1007/s00018-014-1713-1

Bossuyt, P. M., Reitsma, J. B., Bruns, D. E., Gatsonis, C. A., Glasziou, P. P., Irwig, L., et al. (2015). STARD 2015: an Updated List of Essential Items for Reporting Diagnostic Accuracy Studies. BMJ 351, h5527. doi:10.1136/bmj.h5527

Bouchlariotou, S., Liakopoulos, V., Giannopoulou, M., Arampatzis, S., Eleftheriadis, T., Mertens, P. R., et al. (2014). Melatonin Secretion Is Impaired in Women with Preeclampsia and an Abnormal Circadian Blood Pressure Rhythm. Ren. Fail. 36, 1001-1007. doi:10.3109/0886022X.2014.926216

Campos, L. A., Bueno, C., Barcelos, I. P., Halpern, B., Brito, L. C., Amaral, F. G., et al. (2020). Melatonin Therapy Improves Cardiac Autonomic Modulation in Pinealectomized Patients. Front. Endocrinol. 11, 239. doi:10.3389/fendo.2020.00239

Campos, L. A., Cipolla-Neto, J., Amaral, F. G., Michelini, L. C., Bader, M., and Baltatu, O. C. (2013). The Angiotensin-Melatonin axis. Int. J. Hypertens. 2013, 1-7. doi:10.1155/2013/521783

Chen, C., Yang, X., Lei, Y., Zha, Y., Liu, H., Ma, C., et al. (2016). Urinary Biomarkers at the Time of AKI Diagnosis as Predictors of Progression of AKI Among Patients with Acute Cardiorenal Syndrome. Cjasn 11, 1536-1544. doi:10.2215/CJN.00910116 
Chen, G., Zhang, Y., Jin, X., Zhang, L., Zhou, Y., Niu, J., et al. (2011). Urinary Proteomics Analysis for Renal Injury in Hypertensive Disorders of Pregnancy with iTRAQ Labeling and LC-MS/MS. Prot. Clin. Appl. 5, 300-310. doi:10. 1002/prca.201000100

D'agostino, R. (2017). Tests for the normal Distribution. Goodness, 367-420. doi:10.1201/9780203753064-9

Dallal, G. E., and Wilkinson, L. (1986). An Analytic Approximation to the Distribution of Lilliefors's Test Statistic for Normality. Am. Stat. doi:10. 2307/2684607

de Alencar Franco Costa, D., Todiras, M., Campos, L. A., Cipolla-Neto, J., Bader, M., and Baltatu, O. C. (2015). Sex-dependent Differences in Renal Angiotensinogen as an Early Marker of Diabetic Nephropathy. Acta Physiol. (Oxf) 213, 740-746. doi:10.1111/apha.12441

dos Santos, P. A., da Silva, E. R., Vergani, D. d. O. P., Garcia, R. M. R., de Araújo, B. F., et al. (2020). Gestational Diabetes in the Population Served by Brazilian Public Health Care. Prevalence and Risk Factors. Rev. Bras. Ginecol. Obstet. 42, 012-018. doi:10.1055/s-0039-1700797

Farrar, D. (2016). Hyperglycemia in Pregnancy: Prevalence, Impact, and Management Challenges. Ijwh Vol. 8, 519-527. doi:10.2147/IJWH. S102117

Ferreira, A. P. d. S., Szwarcwald, C. L., Damacena, G. N., and Damacena, G. N. (2019). Prevalência e fatores associados da obesidade na população brasileira: estudo com dados aferidos da Pesquisa Nacional de Saúde, 2013. Rev. Bras. Epidemiol. 22, e190024. doi:10.1590/1980-549720190024

Fishel Bartal, M., Lindheimer, M. D., and Sibai, B. M. (2020). Proteinuria During Pregnancy: Definition, Pathophysiology, Methodology, and Clinical Significance. Am. J. Obstet. Gynecol. S0002-9378 (20), S0002-9378(20)309893. doi:10.1016/j.ajog.2020.08.108

Fishel Bartal, M., and Sibai, B. M. (2020). Eclampsia in the 21st century. Am. J. Obstet. Gynecol., S0002-9378(20)31128-5. doi:10.1016/j.ajog.2020. 09.037

Fotheringham, J., Campbell, M. J., Fogarty, D. G., El Nahas, M., and Ellam, T. (2014). Estimated Albumin Excretion Rate versus Urine Albumin-Creatinine Ratio for the Estimation of Measured Albumin Excretion Rate: Derivation and Validation of an Estimated Albumin Excretion Rate Equation. Am. J. Kidney Dis. 63, 405-414. doi:10.1053/j.ajkd.2013.08.009

Galvin, H. K., and DeMuro, P. R. (2020). Developments in Privacy and Data Ownership in Mobile Health Technologies, 2016-2019. Yearb. Med. Inform. 29, 032-043. doi:10.1055/s-0040-1701987

Gathiram, P., and Moodley, J. (2020). The Role of the Renin-AngiotensinAldosterone System in Preeclampsia: a Review. Curr. Hypertens. Rep. 22, 89. doi:10.1007/s11906-020-01098-2

Indarti, J., Susilo, S. A., Hyawicaksono, P., Berguna, J. S. N., Tyagitha, G. A., and Ikhsan, M. (2021). Maternal and Perinatal Outcome of Maternal Obesity at RSCM in 2014-2019. Obstet. Gynecol. Int. 2021, 1-6. doi:10.1155/2021/6039565

Juretzko, A., Steinbach, A., Hannemann, A., Endlich, K., Endlich, N., Friedrich, N., et al. (2017). Urinary Angiotensinogen and Renin Excretion Are Associated with Chronic Kidney Disease. Kidney Blood Press. Res. 42, 145-155. doi:10. $1159 / 000474932$

Kobori, H., and Navar, L. G. (2011). Urinary Angiotensinogen as a Novel Biomarker of Intrarenal Renin-Angiotensin System in Chronic Kidney Disease. Int. Rev. Thromb. 6 (2), 108-116.

Kobori, H., Alper, A. B., Shenava, R., Katsurada, A., Saito, T., Ohashi, N., et al. (2009). Urinary Angiotensinogen as a Novel Biomarker of the Intrarenal ReninAngiotensin System Status in Hypertensive Patients. Hypertension 53, 344-350. doi:10.1161/HYPERTENSIONAHA.108.123802

Lamontagne, A., Côté, A.-M., and Rey, E. (2014). The Urinary Protein-ToCreatinine Ratio in Canadian Women at Risk of Preeclampsia: Does the Time of Day of Testing Matter? J. Obstet. Gynaecol. Can. 36, 303-308. doi:10.1016/S1701-2163(15)30605-8

Leal, L. F., Grandi, S. M., Miranda, V. I. A., Dal Pizzol, T. d. S., Platt, R. W., Silveira, M. F. d., et al. (2020). Hypertensive Disorders of Pregnancy and Medication Use in the 2015 Pelotas (brazil) Birth Cohort Study. Ijerph 17, 8541. doi:10.3390/ ijerph17228541

Lu, H., Cassis, L. A., Kooi, C. W. V., and Daugherty, A. (2016). Structure and Functions of Angiotensinogen. Hypertens. Res. 39, 492-500. doi:10.1038/hr. 2016.17
Mistry, H. D., Kurlak, L. O., Gardner, D. S., Torffvit, O., Hansen, A., Broughton Pipkin, F., et al. (2019). Evidence of Augmented Intrarenal Angiotensinogen Associated with Glomerular Swelling in Gestational Hypertension and Preeclampsia: Clinical Implications. Jaha 8, e012611. doi:10.1161/JAHA.119. 012611

Morris, R. K., Riley, R. D., Doug, M., Deeks, J. J., and Kilby, M. D. (2012). Diagnostic Accuracy of Spot Urinary Protein and Albumin to Creatinine Ratios for Detection of Significant Proteinuria or Adverse Pregnancy Outcome in Patients with Suspected Pre-eclampsia: Systematic Review and Meta-Analysis. BMJ 345, e4342. doi:10.1136/bmj.e4342

Ohashi, N., Ishigaki, S., and Isobe, S. (2019). The Pivotal Role of Melatonin in Ameliorating Chronic Kidney Disease by Suppression of the ReninAngiotensin System in the Kidney. Hypertens. Res. 42, 761-768. doi:10. 1038/s41440-018-0186-2

Phipps, E., Prasanna, D., Brima, W., and Jim, B. (2016). Preeclampsia: Updates in Pathogenesis, Definitions, and Guidelines. Cjasn 11, 1102-1113. doi:10.2215/ CJN.12081115

Pringle, K. G., de Meaultsart, C. C., Weatherall, L. J., Keogh, L., Clausen, D. C., et al. (2018). Urinary Angiotensinogen Excretion in Australian Indigenous and Nonindigenous Pregnant Women. Pregnancy Hypertens. 12, 110-117. doi:10.1016/j. preghy.2018.04.009

Ramiro-Cortijo, D., Herrera, T., Rodríguez-Rodríguez, P., López De Pablo, Á. L., López-Giménez, M. R., Mora-Urda, A. I., et al. (2016). Maternal Plasma Antioxidant Status in the First Trimester of Pregnancy and Development of Obstetric Complications. Placenta 47, 37-45. doi:10.1016/j.placenta.2016. 08.090

Sagrillo-Fagundes, L., Soliman, A., and Vaillancourt, C. (2014). Maternal and Placental Melatonin: Actions and Implication for Successful Pregnancies. Minerva Ginecol 66, 251-266.

Sert, U. Y., and Ozgu-Erdinc, A. S. (2021). Gestational Diabetes Mellitus Screening and Diagnosis. Adv. Exp. Med. Biol. 1307, 231-255. doi:10.1007/ 5584_2020_512

Sousa, M. G., de, Lopes, R. G. C., Rocha, M. L. T. L. F., da, Lippi, U. G., Costa, E., de, S., et al. (2019). Epidemiology of Artherial Hypertension in Pregnants. Einstein (Sao Paulo) 18, eAO4682. doi:10.31744/ einstein_journal/2020ao4682

Stefańska, K., Zieliński, M., Zamkowska, D., Adamski, P., JassemBobowicz, J., Piekarska, K., et al. (2020). Comparisons of Dipstick Test, Urine Protein-To-Creatine Ratio, and Total Protein Measurement for the Diagnosis of Preeclampsia. Ijerph 17, 4195. doi:10.3390/ijerph 17124195

Świątkowska-Stodulska, R., Kmieć, P., Stefańska, K., and Sworczak, K. (2018). Renin-Angiotensin-Aldosterone System in the Pathogenesis of PregnancyInduced Hypertension. Exp. Clin. Endocrinol. Diabetes 126, 362-366. doi:10. 1055/s-0044-102008

Tain, Y.-L., Huang, L.-T., and Hsu, C.-N. (2017). Developmental Programming of Adult Disease: Reprogramming by Melatonin? Ijms 18, 426. doi:10.3390/ ijms 18020426

Tong, S., Kaitu'u-Lino, T. J., Hastie, R., Brownfoot, F., Cluver, C., and Hannan, N. (2020). Pravastatin, Proton-Pump Inhibitors, Metformin, Micronutrients, and Biologics: New Horizons for the Prevention or Treatment of Preeclampsia. Am. J. Obstet. Gynecol. 10.1016/ j.ajog.2020.09.014. doi:10.1016/j.ajog.2020.09.014

Vadalà, C., Cernaro, V., Siligato, R., Granese, R., Laganà, A. S., Buemi, M., et al. (2017). Rischio di Danno Renale a Distanze in Donne Con Preeclampsia [LongTerm Outcome of Renal Function in Women with Preeclampia and Pregestational Diabetes]. G Ital. Nefrol 2017-vol6. (Italian).

Verdonk, K., Visser, W., Van Den Meiracker, A. H., and Danser, A. H. J. (2014). The Renin-Angiotensin-Aldosterone System in Pre-eclampsia: the Delicate Balance between Good and Bad. Clin. Sci. 126, 537-544. doi:10.1042/ CS20130455

Yilmaz, Z., Yildirim, T., Yilmaz, R., Aybal-Kutlugun, A., Altun, B., Kucukozkan, T., et al. (2015). Association between Urinary Angiotensinogen, Hypertension and Proteinuria in Pregnant Women with Preeclampsia. J. Renin Angiotensin Aldosterone Syst. 16, 514-520. doi:10.1177/1470320313510585

Zeng, K., Gao, Y., Wan, J., Tong, M., Lee, A. C., Zhao, M., et al. (2016). The Reduction in Circulating Levels of Melatonin May Be Associated with the 
Development of Preeclampsia. J. Hum. Hypertens. 30, 666-671. doi:10.1038/ jhh.2016.37

Zhang, L., Zhou, Y., Wu, Q., Fan, W., Ye, J., Chen, Y., et al. (2017). Effective Prediction of Preeclampsia by Measuring Serum Angiotensin II, Urinary Angiotensinogen and Urinary Transforming Growth Factor $\beta 1$. Exp. Ther. Med. 14, 391-397. doi:10.3892/etm.2017.4484

Zhang, Y., Sun, C.-M., Hu, X.-Q., and Zhao, Y. (2014). Relationship between Melatonin Receptor 1B and Insulin Receptor Substrate 1 Polymorphisms with Gestational Diabetes Mellitus: a Systematic Review and Meta-Analysis. Sci. Rep. 4, 6113. doi:10.1038/srep06113

Conflict of Interest: The authors declare that the research was conducted in the absence of any commercial or financial relationships that could be construed as a potential conflict of interest.
Publisher's Note: All claims expressed in this article are solely those of the authors and do not necessarily represent those of their affiliated organizations, or those of the publisher, the editors and the reviewers. Any product that may be evaluated in this article, or claim that may be made by its manufacturer, is not guaranteed or endorsed by the publisher.

Copyright (C) 2022 Valias, Gomes, Amaral, Alnuaimi, Monteiro, O’Sullivan, Zangaro, Cipolla-Neto, Acuna, Baltatu and Campos. This is an open-access article distributed under the terms of the Creative Commons Attribution License (CC BY). The use, distribution or reproduction in other forums is permitted, provided the original author(s) and the copyright owner(s) are credited and that the original publication in this journal is cited, in accordance with accepted academic practice. No use, distribution or reproduction is permitted which does not comply with these terms. 